\title{
Vulnerabilidad frente al cambio climático en la Región Metropolitana de Santiago de Chile: posiciones teóricas versus evidencias empíricas
}

Juliane Welz. Helmholtz Centre for Environmental Research (UFZ), Department of Urban and Environmental Sociology, Leipzig, Alemania.

Kerstin Krellenberg. Centre for Environmental Research (UFZ), Department of Urban and Environmental Sociology, Leipzig, Alemania.

RESUmen | Para la Región Metropolitana de Santiago de Chile (RMS), el cambio climático implica diferentes impactos que se suman a la continua expansión urbana y a cambios en los patrones de uso del suelo, los cuales a su vez acrecientan las amenazas hidroclimáticas, como las inundaciones y el calor extremo. En general, estos cambios tendrán notables consecuencias para la vulnerabilidad residencial. Especialmente las familias de bajos recursos y los asentamientos ilegales en grandes ciudades con crecimiento acelerado suelen ser los más vulnerables, debido a una mayor exposición y a una limitada capacidad de respuesta. Este artículo contrasta posiciones teóricas sobre las dimensiones socioeconómicas, físico-constructivas y urbano-rurales de la vulnerabilidad con evidencias empíricas, para entender quiénes son los más afectados por las inundaciones y el calor extremo en la RMs. Como muestran los resultados del estudio, las posiciones teóricas no se reflejan en los análisis cuantitativos. La vulnerabilidad depende de cada contexto y requiere políticas adecuadas para distintos territorios.

PALABRAS CLAVE | vulnerabilidad, riesgos naturales, medioambiente urbano.

ABstract | For the Metropolitan Region of Santiago de Chile (RMS), climate change implies different impacts adding to continuous urban growth and changes in land-use patterns, which in turn reinforce hydro-meteorological hazards such as floods and heat waves. At large, these changes are expected to have significant implications for residents' vulnerability. Particularly, marginalized families living in informal settlements of rapidly growing cities are often highly vulnerable to hazards due to their high exposure and limited coping capacities. This article seeks to contrast theoretical assumptions regarding the socioeconomic, the physicalstructural and the urban-rural dimensions of vulnerability with empirical data in order to illuminate what groups are likely to be affected. As the research results show, general theoretical arguments are not reflected in the empirical data analysis. In contrast, residents' vulnerability to climate change is context-specific and requires appropriate policy strategies for differing areas.

KEYwORDS | vulnerability, natural risks, urban environment. 


\section{La vulnerabilidad al cambio climático: el caso específico de la Región Metropolitana de Santiago}

Las ciudades de América Latina, igual que otras ciudades en todo el mundo, se enfrentan a una variedad de desafíos ambientales y ecológicos que conllevan impactos significativos en los sectores de la salud, la producción y la economía, así como en la pobreza y desigualdad (Jordán, Rehner \& Samaniego, 2012). A esto se sumarán los impactos del cambio climático previstos para la región, que exacerbarán estos desafíos (Winchester \& Szalachman, 2012).

Según todos los pronósticos, la Región Metropolitana de Santiago de Chile (RMs) se verá afectada por el cambio climático en las próximas décadas con una disminución de las tasas anuales medias de precipitaciones y un aumento de las temperaturas medias (Cortés et al., 2012). En combinación con el acelerado crecimiento urbano y la pérdida de la función de los suelos de retención y enfriamiento que cumple la vegetación en las áreas urbanas y suburbanas (Müller \& Höfer, 2014), este cambio implica varios efectos. Entre otros, el aumento del número de inundaciones y de los días de calor extremo, especialmente en zonas densamente urbanizadas. A pesar de que hoy en día la precipitación media ya es comparativamente baja, las inundaciones se producen durante los meses de invierno en días con precipitaciones intensas, interrumpiendo en algunas instancias el funcionamiento urbano y dejando daños estructurales en las calles y en las plantas bajas de las viviendas. En general se producen pérdidas materiales, pero no existe una amenaza a la vida humana, ya que el nivel de las inundaciones es mesurado (Krellenberg, Müller, Schwarz, Höfer \& Welz, 2013). El calor extremo, en cambio, es y será un fenómeno frecuente en el periodo de verano, que afecta principalmente la salud y el bienestar de la población residente en áreas con escasa cobertura vegetal o en construcciones con aislamiento insuficiente (Bell et al., 2008; Lafortezza, Carrus, Sanesi \& Davies, 2009). También se puede asumir que el cambio climático ocasiona efectos crónicos de mediano a largo plazo, como la gestión de la escasez y el abastecimiento de los (escasos) recursos hídricos, que incidirán en la vida cotidiana al igual que en las actividades económicas de la metrópoli y sus alrededores (Swyngedouw, Kaïka \& Castro, 2002; Domènech, Supranamiam \& Saurí, 2010).

Teniendo en cuenta este contexto, el presente artículo indaga en las áreas expuestas a inundaciones y al calor extremo dentro de la RMS e investiga los diferentes patrones espaciales de vulnerabilidad. De este modo, se busca respuestas a las siguientes preguntas: ¿de qué manera la RMS es vulnerable frente a estos acontecimientos climáticos? ¿Quién se verá afectado y por qué?

Antes de responder a estas preguntas, es de gran importancia aclarar el término "vulnerabilidad". En América Latina, varios autores se han referido al concepto de la vulnerabilidad, en la mayoría de los casos a la vulnerabilidad social, que está vinculada a la condición de pobreza y que se ha convertido en un rasgo social dominante (Pizarro, 2001). Así, "el aumento de la vulnerabilidad, causada por las contingencias y presiones de la economía y de la vida actual -y las dificultades para enfrentarlas- es una característica de los pobres de la región" (Pizarro Hofer, 1999, p. 20). Sin embargo, la vulnerabilidad no solamente está asociada a las condiciones 
de un individuo, sino que también está influenciada por las condiciones del entorno en el que se inserta su vida cotidiana. Así, ser vulnerable implica un cierto grado de fragilidad, una situación de amenaza o de mayor susceptibilidad a los dańos (Feito, 2007). Esto también se ha discutido en relación con los desastres naturales donde se han producido espacios de vulnerabilidad o de exposición de las personas a situaciones de amenazas, generando condiciones de desprotección.

En referencia al cambio climático, existe una amplia gama de conceptos de vulnerabilidad, referidos principalmente a las situaciones de marginalidad, susceptibilidad, adaptabilidad, fragilidad y riesgo (Leichenko \& O’Brien, 2002). Se distinguen generalmente dos nociones de vulnerabilidad: vulnerabilidad como resultado (outcome vulnerability) y vulnerabilidad contextual (contextual vulnerability) (O'Brien, Eriksen, Nygaard \& Schjolden, 2007; Heinrichs, Krellenberg \& Fragkias, 2013). La primera noción se centra en el sistema natural y considera los efectos adversos del cambio climático, los cuales aumentan la probabilidad de peligro y de exposición de sistemas y sectores. Esta aproximación considera la vulnerabilidad como el punto final de una secuencia de análisis y aplica una perspectiva de arriba a abajo (O’Brien et al., 2007; Magrin et al., 2007; Adger et al., 2007). La segunda noción, que se adopta en este artículo, relaciona la vulnerabilidad con las condiciones humanas, para lo cual considera la forma en que la sociedad es vulnerable al cambio climático y cómo se adapta a él, enmarcando tal proceso en el contexto de "equidad" (Adger $\&$ Kelly, 1999; Chambers, 1989). En último término, la condición de vulnerabilidad se conceptualiza como resultado de las estructuras y los procesos ambientales, sociales, culturales, institucionales y económicos, aproximación que hoy lleva a relacionarla con una distribución desigual de los recursos (Adger \& Kelly, 1999; Pizarro, 2001), producto de las complejas interrelaciones entre lo espacial y lo social, así como entre lo global y lo local. Estas interrelaciones influyen de una u otra manera en la sensibilidad de los lugares y de las poblaciones al cambio climático, y también en su capacidad de responder a los desastres naturales (O'Brien et al., 2007).

A partir de lo anterior, con el término vulnerabilidad nos referimos a una condición humana provocada por la exposición de las personas a peligros, condición que está estrechamente asociada a la situación social y socioeconómica de los afectados (Wisner, Blaikie, Cannon \& Davis, 2004; Cutter, Mitchell \& Scott, 2000; Pelling, 1999). Siguiendo a Wisner et al. (2004), se entiende la vulnerabilidad como la habilidad de una persona o cierto grupo para generar una capacidad de anticipar, superar, resistir y recuperarse de un impacto. En este sentido, se distinguen tres componentes de la vulnerabilidad: exposición, susceptibilidad y capacidad de recuperación/respuesta (véase, por ej., Kuhlicke, Kabisch, Krellenberg \& Steinführer, 2012; Romero-Lankao, 2010; Wisner et al., 2004; McCarthy, Canziani, Leary, Dokken \& White, 2001).

- La exposición se ve como la precondición física a verse afectado por el impacto de una amenaza proveniente de un acontecimiento natural (Fuchs, Kuhlicke \& Meyer, 2011). La exposición a amenazas es un "external stress placed on [people's] livelihoods and well-being” (Adger \& Kelly, 1999, p. 253). 
- La susceptibilidad describe la precondición a sufrir un daño debido a las condiciones residenciales desventajosas (Birkmann et al., 2013), como una infraestructura inadecuada, la falta de acceso a los servicios básicos o ciertos materiales de construcción "inestables" (Kuhlicke, Scolobig, Tapsell, Steinführer \& de Marchi, 2011).

- La capacidad de recuperación/respuesta se refiere a la virtud de superar y recuperarse del impacto de una amenaza a corto plazo, como también a estar prevenido ante esta (Wisner et al., 2004). La capacidad de recuperación está directamente relacionada con el acceso de las personas a recursos cruciales (por ej. empleo, educación, incorporación en programas de manejo del riesgo de desastres, redes sociales), los cuales hacen posible que las personas superen los riesgos (Kaztman \& Filgueira, 1999).

Partiendo del supuesto de que las condiciones de las vulnerabilidades existentes en un área segregada, desigual y que sufre alteraciones en los usos del suelo como resultado de la expansión urbana -que es el caso de la RMs (Heinrichs, Krellenberg, Hansjürgens \& Martínez, 2011)-, surge la siguiente pregunta: ¿cuáles son los grupos vulnerables a los impactos del cambio climático, y por qué? Siguiendo estas interrogantes, el artículo indaga en tres dimensiones que los estudios tratan frecuentemente en cuanto a la distribución espacial de las vulnerabilidades y en relación con los impactos del cambio climático: i) la dimensión socioeconómica; ii) la dimensión físico-constructiva, y iii) la dimensión urbano-rural. Se parte del supuesto de que estas tres dimensiones tienen influencia en las vulnerabilidades en cuanto a su exposición, susceptibilidad y/o capacidad de recuperación frente al peligro de inundaciones y de calor extremo.

a) La dimensión socioeconómica. Una característica de las ciudades crecientes es que los grupos de bajos recursos viven en terrenos de situación adversa (por ej. en sitios alejados, vertederos, orillas del río) a causa de un acceso desigual al suelo o a los mercados de la vivienda (Ward, 1998; Kantor \& Nair, 2005; Pelling, 2002). De esta manera, la dimensión socioeconómica está directamente vinculada a los procesos de expansión urbana y a la segregación socioespacial, algo muy característico de las ciudades de América Latina. De allí que se podría asumir que la vulnerabilidad a los peligros -como, por ej., las inundaciones y el calor extremo- se expresará de forma desigual tanto en cuanto a la exposición como a la capacidad de recuperación (Cutter, Boruff \& Shirley, 2003). Se supone que las familias de altos ingresos tendrán más facilidades para protegerse (Hardoy \& Pandiella, 2009) y mayor rapidez para recuperarse de las pérdidas, debido al acceso a seguros, redes y programas de seguridad social (Uitto, 1998; Cutter et al., 2000; Burton, Kates \& White, 1993; Wisner et al., 2004; Hewitt, 1997; Puente, 1999).

b) La dimensión físico-constructiva. Esta dimensión está vinculada con la dimensión previamente presentada, en el sentido de que la situación socioeconómica influye en la calidad y la condición física de las viviendas. Se supone que las 
familias de bajos recursos habitan viviendas precarias, que no cuentan con construcciones efectivas para protegerse de los peligros (Wisner, 1998; Cutter et al., 2000) o viceversa, las viviendas de la población acomodada están mejor protegidas. Así, la dimensión físico-constructiva influye en los daños potenciales y su recuperación, y está vinculada con la "susceptibilidad".

c) La dimensión urbano-rural. Generalmente, se presume que la población urbana muestra una vulnerabilidad distinta que la población rural, debido a los diferentes ingresos promedio del hogar, la dependencia de las economías respecto del sector primario y de las actividades agrícolas (Cutter et al., 2003). Mientras que en las áreas rurales el sector primario se verá fuertemente afectado por los efectos del cambio climático (Magrin et al., 2007), hay menos claridad sobre las diferencias entre la exposición de la población rural y la urbana frente a la amenaza por inundación y calor extremo. Pero se supone que en las grandes áreas urbanas con dependencias rurales, tal como la RMs, hay una diferenciación significativa.

Estos tres fundamentos constituyen la base central de este artículo y serán comprobados por un análisis de vulnerabilidad frente a la amenaza por inundación y calor extremo, que ya ha sido experimentada por la población de la RMS en la actualidad, y que se estima que se intensificará en las próximas décadas (Krellenberg et al., 2013). En este sentido, la RMS resulta ser un caso interesante para discutir posiciones teóricas versus evidencias empíricas sobre la vulnerabilidad frente al cambio climático.

\section{Enfoque metodológico}

Siguiendo el enfoque de la vulnerabilidad contextual, el presente artículo enfatiza la exposición a la amenaza por inundación y calor extremo para las personas, los hogares y las viviendas. Se adopta una metodología deductiva en el análisis de vulnerabilidad (véase O'Brien et al., 2004), utilizando datos cuantitativos del Censo Nacional de Población y Vivienda 2002 en términos socioeconómicos, físico-constructivos y urbano-rurales (INE, 2002). Este análisis considera la totalidad de la RMS, la cual está compuesta por 52 comunas (véase gráfico 1); se utiliza para los cálculos estadísticos el nivel de manzana, que representa la unidad espacial más pequeña. Se aplicó a las áreas de inundaciones derivadas de Ayala, Espinoza y Saragoni (1987), que forman parte del Plan Regulador Metropolitano de Santiago (PRMs). En cambio, los datos de la distribución espacial del calor extremo (promedio de la temperatura de superficie $>35^{\circ} \mathrm{C}$ ) provienen de Höfer (2013) y se basan en el análisis de las imágenes Landsat 5 TM con una resolución geométrica de $120 \mathrm{~m}$, estimando la temperatura de superficie del área urbana edificada (véase también Krellenberg et al., 2013).

Las áreas de inundaciones y de calor extremo fueron superpuestas con los datos censales. Para determinar las dimensiones socioeconómicas y físico-constructivas en la vulnerabilidad se emplean dos tipos de índices: el índice GSE y el índice COFIVI. El primero - "índice de grupos socioeconómicos” (GSE) - es un índice ampliamente 
utilizado en Chile para identificar la situación socioeconómica de los hogares. Dado que los ingresos no forman parte del censo, el índice GSE se refiere a niveles de educación del jefe de hogar y a la posesión de diez bienes. Clasificando estos parámetros, se distinguen cinco clases $(\mathrm{ABC} 1, \mathrm{C} 2, \mathrm{C} 3, \mathrm{D}, \mathrm{E}) . \mathrm{ABC} 1$ se refiere al estrato socioeconómico más alto. Para el presente estudio, el índice se calculó para la RMS aplicando la metodología de Sabatini, Salcedo, Wormald y Cáceres (2010) y Welz (2014). Dado que el índice GSE presenta numerosas limitantes (Ruiz-Tagle \& López, 2014), se propone un segundo índice -índice COFIVI-, que se refiere a las condiciones físicas de las viviendas, incluyendo los materiales de construcción para el techo, las paredes y el piso, así como el acceso al agua potable y el tipo de saneamiento (véase Krellenberg et al., 2013). Este índice brinda información sobre cinco grupos de condiciones físicas de vivienda (en las cuales la categoría V representa la calidad más baja y la categoría I la más alta) (Welz, Schwarz \& Krellenberg, 2014).

\section{GRÁFICO I | La RMS y áreas de inundación y calor extremo}

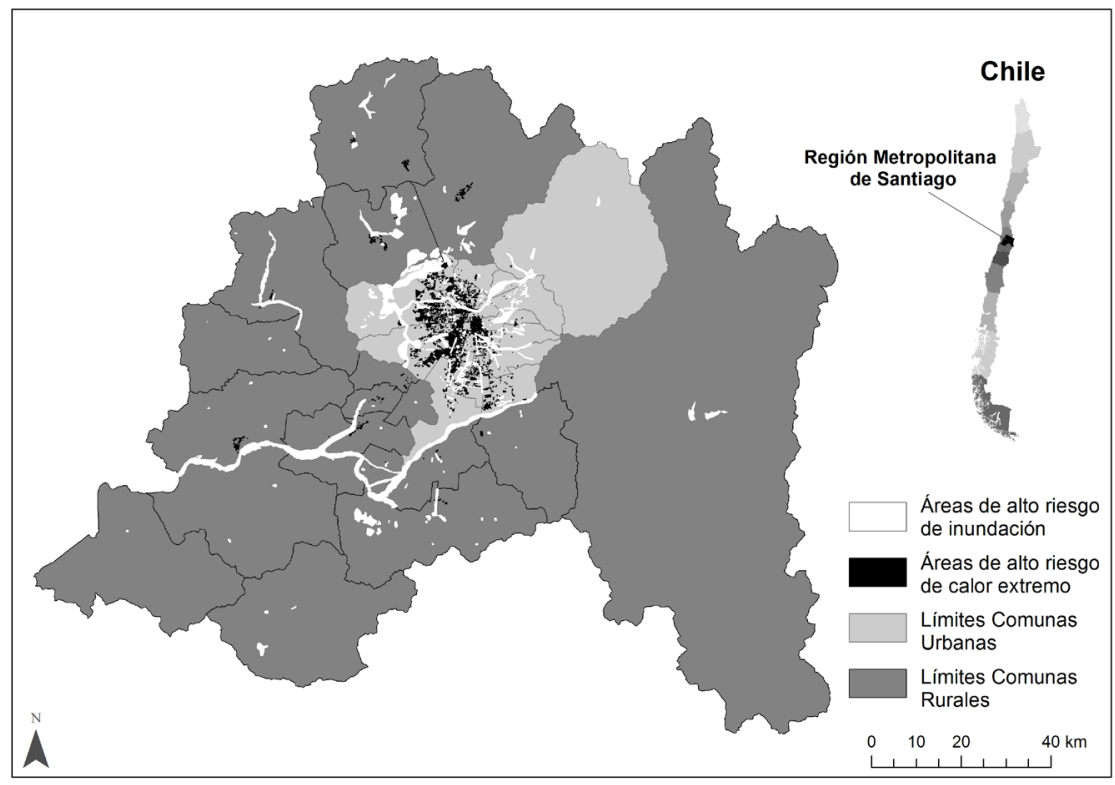

FUENTE ELABORACIÓN PROPIA A PARTIR DE DATOS DEL INE (2002), MÜLLER Y HÖFER (2OI4)

Para evaluar los patrones de desigualdad en la distribución de la exposición a amenazas, se utilizó una versión modificada del "indicador comparativo de riesgo medioambiental" (Comparative Environmental Risk Indicator, CERI) de Walker et al. (2006). Este indicador emplea un cociente (la proporción de tasas) y se calcula para cada grupo de los índices GSE y COFIVI en relación con los otros grupos, considerando el nivel regional y la amenaza (inundación o calor extremo). Existe una alta probabilidad de exposición cuando los valores son mayores a 0 , mientras que un 
valor menor a 0 representa una probabilidad más baja que los demás grupos (véase también Welz et al., 2014). Los pasos metodológicos se presentan en el gráfico 2.

\section{GRÁfICO 2 | Metodología aplicada}

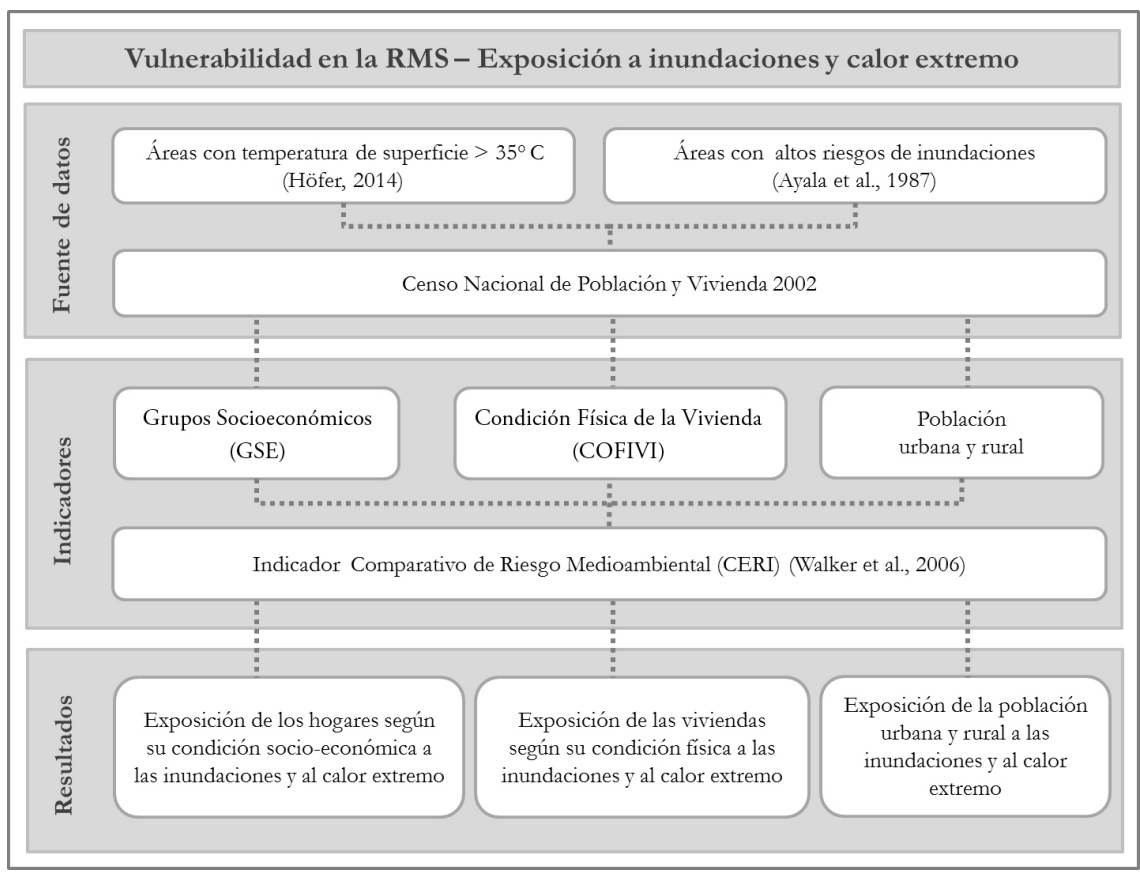

FUENTE ELABORACIÓN PROPIA

\section{La dimensión socioeconómica en los patrones de distribución de la vulnerabilidad}

La vulnerabilidad frente a la amenaza por inundación y calor extremo depende, en primer lugar, de la exposición a la respectiva amenaza. En las grandes ciudades caracterizadas por una acelerada expansión, los procesos de transformación implican, entre otras cosas, cambios en el sistema natural urbano y en los factores sociales (Kuhlicke et al., 2012) que determinan la vulnerabilidad a la amenaza. Así, tanto la amenaza, como su cualidad física y la condición socioeconómica de los hogares, interactúan con el tejido social del lugar (Cutter et al., 2000). En general, en el mundo académico hay consenso sobre la evidencia de que las personas con ciertas características sociales están más expuestas que otras, especialmente aquellas que carecen de acceso a la información, a los servicios, a los recursos financieros o al apoyo social (Wisner, 1998; Adger \& Kelly, 1999; Cutter et al., 2000). Cuando se superponen estas carencias con sucesos peligrosos en un lugar específico, la población sufre más dańos en el momento inmediato o es menos capaz de recuperarse posteriormente (Wisner, 1998). Por eso, Adger y Kelly (1999) constatan que la 
dimensión socioeconómica del hogar es un aspecto importante en la vulnerabilidad residencial, debido a su relación directa con el acceso a los recursos. Según los autores, la pobreza es un factor clave, ya que está directamente relacionada con la marginalidad y la falta de acceso a los recursos y a la información, y afecta la capacidad de recuperación, supervivencia y resistencia frente a un suceso peligroso.

En la misma línea argumentan Winchester y Szalachman (2012) para las ciudades latinoamericanas, donde la vulnerabilidad no es solo una dimensión fundamental de la pobreza, sino que más bien ambas van de la mano. Argumentan que los habitantes particularmente vulnerables a los impactos del cambio climático son aquellos que carecen de recursos económicos debido a la precariedad laboral y educacional y que viven en áreas de alto riesgo de sucesos peligrosos (Hardoy \& Pandiella, 2009). Aún más, la pobreza urbana tiene una connotación espacial, que se expresa en un patrón de segregación residencial tradicional. Esta es una característica específica de las ciudades de América Latina (Sabatini, Cáceres \& Cerda, 2001). No obstante, en el curso de los últimos años se ha observado cambios en el patrón de segregación residencial en las ciudades latinoamericanas, entre otras en la RMs, donde la escala espacial de segregación se ha reducido (Cáceres \& Sabatini, 2004; González \& Rodríguez, 2006). Las políticas de liberalización de los mercados de suelo y los procesos de globalización económica y cultural han impulsado un aumento en la construcción de condominios o barrios cerrados en áreas urbanas donde anteriormente residían familias de bajos recursos (Hidalgo, 2004; Janoschka, 2002; Sabatini, 2000; Welz, 2014). Estas nuevas viviendas suelen estar localizadas en un medioambiente dinámico, como el piedemonte andino de la RMs. Debido a la continua urbanización en esa zona, la infiltración de las aguas de lluvia ya no está garantizada y la existencia de inundaciones y aluviones ha causado pérdida de vidas, la destrucción de la infraestructura y de viviendas, así como interrupciones en la circulación y la vida normal de la metrópoli (Romero \& Vásquez, 2005).

Ante este contexto, la tesis de que los grupos de escasos recursos son los más vulnerables frente a los impactos del cambio climático debería ser nuevamente comprobada, en particular para la RMs. Revisando los datos censales para la RMS, se determinó aproximadamente 165.000 hogares con exposición a inundaciones y cerca de 210.000 hogares con exposición al calor extremo en el año 2002. Considerando la situación socioeconómica de estos hogares mediante el índice de GSE, se puede indagar en la dimensión socioeconómica de la vulnerabilidad de la RMs. Al analizar la distribución del número total de los hogares expuestos a inundaciones y al calor extremo, según este índice salta a la vista el GSE D (estrato bajo), que representa el número total más alto tanto de exposición a inundaciones $(55.540$ hogares) como al calor extremo (94.580 hogares) (gráfico 3). Sin embargo, si se tiene en cuenta el porcentaje de cada grupo socioeconómico, el resultado es diferente. La exposición a inundaciones muestra una distribución relativamente igualitaria dentro de los GSE, con el porcentaje más alto en el GSE ABC1 (estrato alto) de un $14,4 \%$, diferente a la exposición al calor extremo, donde el GSE E (estrato muy bajo) revela el porcentaje más alto, con un 19,0\% (gráfico 3).

Aplicando el indicador CERI, esta probabilidad diferenciada de la exposición a inundaciones y al calor extremo en la RMs está fundamentada. La probabilidad de 
exposición a inundaciones es muy elevada para el ABC1 (36,7\%), mientras que el C3 $(-11,4 \%)$ y el D $(-6,7 \%)$ se caracterizan por una baja probabilidad. La probabilidad de exposición al calor extremo en la RMs marca una tendencia inversa a la exposición a inundaciones. El gráfico 4 muestra claramente que los GSE D y E (estratos bajos) tienen una probabilidad mayor a $40 \%$ que los demás grupos en el caso de exposición a calor extremo. Al contrario, en este aspecto los grupos socioeconómicos más altos tienen una probabilidad significativamente menor, especialmente el $\mathrm{ABC} 1 \mathrm{con}$ un $-77,3 \%$. El gráfico 4 resume los resultados para el índice GSE.

GRÁFICO 3 | Exposición a inundaciones y calor extremo según GSE, RMS, 2002

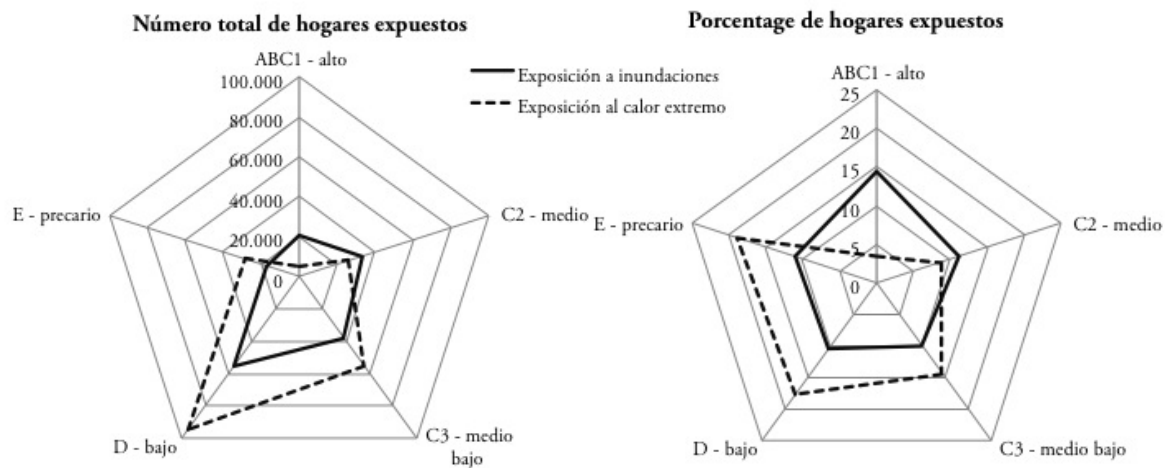

FUENTE ELABORACión PROPIA A PARTIR DE DATOS DEL INE (2002), MÜLLER Y HÖFER (2OI4)

GRÁfICO 4 | Porcentaje de probabilidad de exposición (CERI) a inundaciones y calor extremo por estrato socioeconómico (índice GSE), RMS, 2002

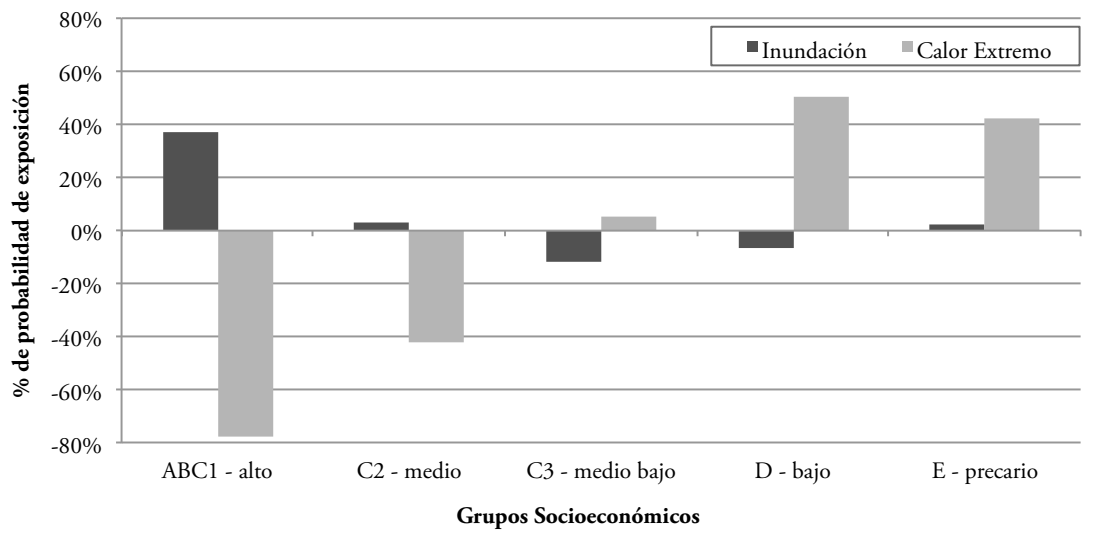

FUENTE Elaboración PROPIA A PARTIR DE DATOS DEL INE (2002), MÜLLER Y HÖFER (2OI4)

Estos resultados desafían claramente la tesis de que los grupos de escasos recursos sean los más vulnerables ante los impactos del cambio climático en términos de la exposición a peligros, e indican que la dimensión socioeconómica de la 
vulnerabilidad no retrocede ante un grupo socioeconómico específico. Como han mostrado los resultados para la RMs, las familias acomodadas son de igual modo vulnerables al cambio climático por su lugar de residencia en zonas de alto riesgo de inundaciones. Apoyando a Eriksen y O'Brien (2007), la pobreza y la vulnerabilidad al cambio climático no son problemas idénticos que se pueden relacionar de forma automática. Además, su vulnerabilidad se produce a diferentes niveles y es el resultado de diferentes patrones que dependen de sus estilos de vida, relaciones sociales y de sus respectivos modelos de estrategias para adaptarse y recuperarse del peligro.

\section{La dimensión físico-constructiva en los patrones de distribución de la vulnerabilidad}

Las proyecciones estadísticas para la RMs señalan un crecimiento poblacional mayor de ocho millones de habitantes para el año 2030 (Ministerio de Vivienda y Urbanismo [MINVU], 2008). Este crecimiento demográfico generará una demanda de vivienda en la región que resultará en transformaciones espaciales y funcionales en el uso del suelo, en particular en el cambio de áreas no urbanas (áreas agrícolas, bosques naturales o humedales) en áreas urbanas (por ej., de usos residenciales, industriales y/o de servicios) (Banzhaf et al., 2012). En conjunto, con estos cambios ya se observa la pérdida de servicios ambientales, tales como la infiltración de aguas pluviales, la mitigación de islas de calor y la conservación de la biodiversidad en algunas zonas de la ciudad (Ebert, Welz, Heinrichs, Krellenberg \& Hansjürgens, 2010; Krellenberg et al., 2013). Estas tendencias influyen en la cantidad e intensidad de los peligros de inundaciones, calor extremo y/o aluviones (Romero, Moscoso \& Smith, 2009) y, a la par, se incrementa la exposición de los habitantes a dichas amenazas.

En términos generales, la acelerada expansión urbana y sus dinámicas en el sector residencial contribuyen a la generación de amenazas causadas por construcciones residenciales de baja calidad y localización arbitraria en zonas de alto riesgo ambiental (Uitto, 1998). Especialmente las viviendas informales habitadas por familias de bajos recursos se relacionan con una mayor vulnerabilidad (Wisner, 1998; Cutter et al., 2000). Al no tener alternativa, los grupos socioeconómicos precarios -también en otras ciudades de América Latina- tienden a asentarse en zonas de alto riesgo ambiental y construyen sus viviendas con materiales precarios (Hardoy \& Pandiella, 2009; Winchester \& Szalachman, 2012). Estas viviendas no suelen cumplir con las normas oficiales de construcción y se caracterizan por altos niveles de hacinamiento y falta de mantenimiento (Winchester, 2008). La mayoría de las viviendas carece de un sistema de aire acondicionado o aislamiento adecuado que impida el paso de la humedad y el frío en la temporada de invierno y del calor durante el verano (Hardoy \& Pandiella, 2009).

Contemplando el índice COFIVI para la RMS, se puede indagar en la condición físico-constructiva de los patrones espaciales de la vulnerabilidad. Según este índice, en total, el 10,9\% de las viviendas (aprox. 152.000) se vio expuesto a inundaciones, y el $13,7 \%$ de las viviendas (aprox. 190.000) estuvo afectado por el calor extremo en la RMs en el año 2002 (Krellenberg et al., 2013). Según los cálculos, se observan 
patrones de exposición similares a la dimensión socioeconómica: todas las viviendas en la RMS están expuestas a inundaciones y al calor extremo, independientemente de su condición física. En números totales, la categoría Iv (deficitaria) cuenta con los valores más altos de exposición a inundaciones (44.636 viviendas) y al calor extremo (70.116 viviendas) (gráfico 5) o, expresado de otra manera, aproximadamente una de cada tres viviendas expuestas a inundaciones o al calor extremo presenta condiciones físicas deficitarias. Sin embargo, teniendo en cuenta el porcentaje de cada categoría, el resultado es diferente. La exposición a inundaciones muestra una distribución relativamente igualitaria dentro de las categorías, con el porcentaje más alto en las viviendas con excelentes condiciones físicas (COFIVI I), de un 13,0\%, diferente a la exposición al calor extremo, donde la categoría $\mathrm{v}$ (precaria) revela el porcentaje más alto, con un 20,1\% (gráfico 5).

GRÁfICO 5 | Exposición a inundaciones y al calor extremo según COFIVI, RMS, 2002

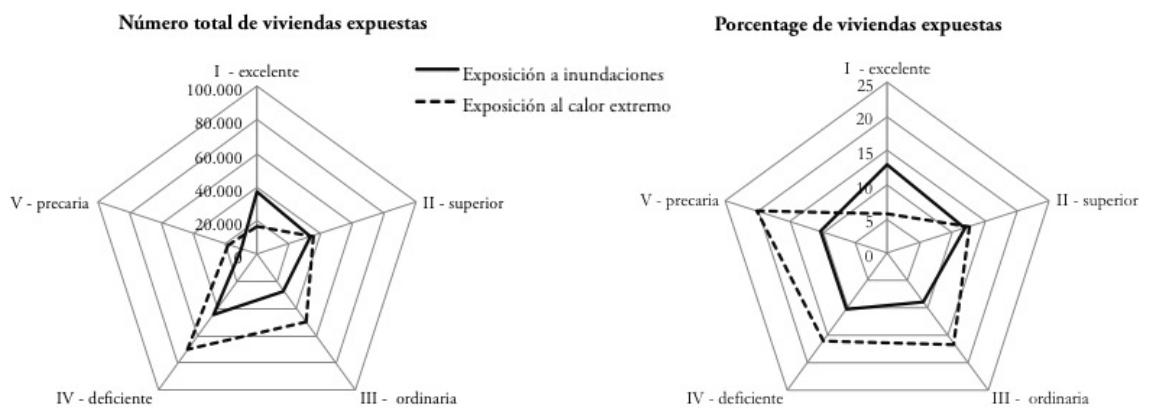

FUENTE ELABORACión PROPIA A PARTIR DE DATOS DEL INE (2002), MÜLLER Y HÖFER (2OI4)

Esta conclusión también está respaldada por los resultados del indicador CERI. La probabilidad de exposición a inundaciones es un 26,0\% más alta para la categoría COFIVI-I (excelente) y un 13,0\% más alta para la categoría Cofivi-II (alta) en comparación con los demás grupos (gráfico 6). La probabilidad de exposición al calor extremo en la RMS marca un patrón inverso: cuanto más baja (deficitaria, precaria) es la categoría COFIVI, más alta es la probabilidad de que se ubique en zonas expuestas al calor extremo. El gráfico 6 muestra claramente que las viviendas de condiciones físicas precarias (COFIVI-v) tienen una probabilidad superior a 50\% de exposición al calor extremo, mayor que la de los demás grupos. En cambio, las viviendas con excelentes condiciones físicas (COFIVI-I) tienen una probabilidad de un $-63,2 \%$ de exposición al calor extremo, significativamente menor que los demás grupos.

Para formular políticas de adaptación, es fundamental reconocer que es más probable que las viviendas de alta calidad se encuentren en áreas de inundación, lo que difiere del caso de exposición al calor extremo. Para este último caso, las viviendas con condiciones físicas deficientes o de baja calidad son las que muestran una elevada probabilidad de exposición. 


\section{GRÁfICo 6 | Porcentaje de probabilidad de exposición (CERI) a inundaciones y al calor extremo por condición física de la vivienda (índice cofivi), RMS, 2002}

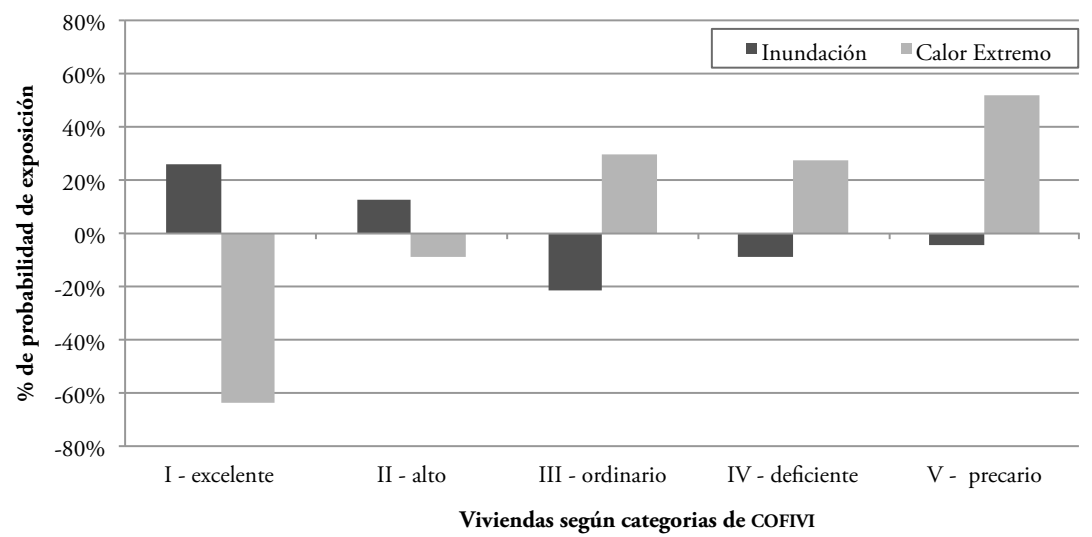

FUENTE ELABORACIÓN PROPIA A PARTIR DE DATOS DEL INE (2002), MÜLLER Y HÖFER (2OI4)

\section{La dimensión urbano-rural en los patrones espaciales de la vulnerabilidad}

El cambio climático tendrá implicaciones tanto en las áreas urbanas como en las rurales. Revisando la bibliografía internacional, se advierte una tendencia hacia el argumento de que las áreas rurales son más vulnerables en comparación con las áreas urbanas, debido a la dependencia de las economías locales respecto del sector primario (Cutter et al., 2000; Cross, 2001; Leichenko \& O’Brien, 2002). Siguiendo a Cross (2001), la economía rural es altamente dependiente del sector agrícola, el cual es sensible a sucesos climáticos como sequías, inundaciones, heladas y granizadas. Así, estos sucesos pueden resultar perjudiciales para los agricultores. Los cultivos y la población rural se ven afectados por el aumento de los precios al consumidor (Mysiak et al., 2012) y por las pérdidas en la cosecha y en ingresos. Estos cambios exponen a la población rural a condiciones muchas veces desconocidas (Leichenko \& O’Brien, 2002) y estrechamente relacionadas con la infraestructura básica (Cross, 2001). Además, el área rural se ve afectada por cambios en el uso del suelo -particularmente en la periferia urbana- producidos por la deforestación, el aumento de la impermeabilidad y la erosión de los suelos, y que promueven inundaciones, islas de calor y deslizamientos (Cross, 2001; Krellenberg et al., 2013; Müller \& Höfer, 2014). Por ello, la vulnerabilidad de las áreas rurales debe ser entendida desde una perspectiva integrada y polifacética (Leichenko \& O’Brien, 2002), la cual exige un análisis que integre una amplia variedad de indicadores de vulnerabilidad.

Una primera aproximación a la temática que indague en la dimensión urbanorural en la vulnerabilidad a través del análisis cuantitativo de la población afectada por inundaciones y el calor extremo en ambas áreas, no permite conclusiones sobre las posibles implicaciones para el sector agrícola. Es decir, no se evaluarán los impactos del cambio climático sobre el sector primario. Consecuentemente, 
revisando los datos de la RMs, se destacaron cambios en el uso del suelo entre los años 2001, 2005 y 2009. Según Müller y Höfer (2014), el crecimiento de las áreas edificadas ha sido muy alto en el período entre 2001 y 2005 en comunas rurales como Colina (455 ha), Lampa (333 ha), Buin (263 ha) y Talagante (212 ha). Entre los años 2005 y 2009, la expansión urbana seguía en las comunas de Lampa (1077 ha) y Colina (142 ha). Esta expansión urbana ha ido acompańada por una reducción de áreas verdes y de zonas agrícolas que ha resultado en cambios en la cobertura vegetal de las comunas rurales de la RMs entre los ańos 2001 y 2009 (Müller \& Höfer, 2014). El impacto de la expansión urbana es especialmente alto en las comunas de la periferia urbana. Un ejemplo es la comuna de Lampa, que ha sufrido pérdidas de áreas verdes de 400 ha y de áreas agrícolas de 740 ha entre 2001 y 2005, y otras 302 ha de áreas verdes entre 2005 y 2009. Otras comunas rurales, como Colina, Calera de Tango, María Pinto y Pirque, se caracterizan por una disminución de áreas agrícolas entre 201 ha y 570 ha. Siguiendo a Müller \& Höfer (2014), es muy probable que la expansión urbana y el cambio de los patrones del uso de suelo fortalezcan el aumento de las amenazas y expongan a personas, hogares y viviendas a inundaciones y al calor extremo, tanto en áreas rurales como urbanas.

Ya en 2002, aproximadamente 610.000 personas en la RMs fueron expuestas a inundaciones (11,1\%) y 666.000 personas (12,1\%) al calor extremo (gráfico 7). Incluyendo la dimensión urbano-rural en la distribución de estas amenazas, es evidente que hay algunas diferencias. La exposición a inundaciones, según datos disponibles, es claramente sobresaliente para la población urbana que está más afectada, tanto en cifras absolutas como relativas. Aquí sobresalen las comunas de Vitacura (34\%), Lo Barnechea (30\%) y La Reina (28\%), todas localizadas en el piedemonte andino. Estas comunas han experimentado un importante aumento en las superficies construidas entre 2001 y 2005. Vitacura incrementó su área edificada en 255 ha, Lo Barnechea en 399 ha y La Reina en 214 ha (Müller, 2012). La pérdida de áreas de retención y el aumento de superficies impermeables ha llevado a altas cifras de personas expuestas a inundaciones.

El calor extremo, en cambio, afecta con un 15,1\% mayoritariamente a la población rural, si se consideran las cifras relativas (gráfico 7). Destacan las comunas de Lampa (27\%), Colina (26\%) y Talagante (16\%). En particular las comunas de Lampa y Colina han experimentado un cambio profundo en el uso del suelo, parecido al que se ha observado para las comunas del piedemonte andino. Aquí, igual que en otras áreas de la RMs, la expansión urbana ha llevado al sellado de varias superficies agrícolas con nuevos desarrollos urbanos y proyectos inmobiliarios. En el caso de las áreas rurales, es muy probable que estos cambios en el uso del suelo hayan promovido un aumento del calor extremo en estas áreas.

Resumiendo, el alto nivel de expansión espacial de las áreas urbanas en la RMS ha dado lugar a importantes cambios en el uso del suelo, entre ellos la pérdida de áreas verdes o naturales y de zonas agrícolas para ser usadas como nuevas zonas residenciales y para infraestructuras de transporte, de servicios y de comercio. Esto lleva a una perturbación de los ecosistemas y a un aumento de las amenazas por inundación y de calor extremo. Los resultados indican la necesidad de adaptar tanto las áreas urbanas como las rurales a los efectos del cambio climático, y llaman a 
elaborar medidas específicas de adaptación para las áreas rurales y urbanas. Como dice Bankoff (2001), los asentamientos rurales no son "less at risk than their urban counterparts" (p. 26). Resumiendo, seguimos el argumento de Mitchell (1999), que considera que la predominancia de sucesos climáticos en áreas rurales oculta el hecho de que las áreas urbanas están surgiendo como los principales lugares de desastres naturales.

GRÁfICO 7 | Exposición a inundaciones y al calor extremo según el área urbanorural, RMs, 2002

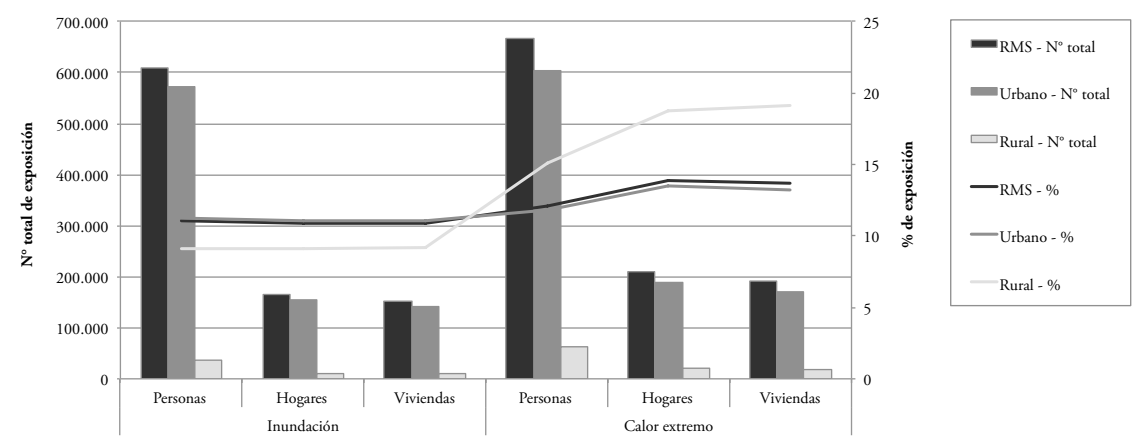

FUENTE ELABORACIÓN PROPIA A PARTIR DE DATOS DEL INE (2002), MÜLLER Y HÖFER (20I4)

\section{Lecciones para la adaptación de la RMS al cambio climático}

Los resultados obtenidos y discutidos en el presente artículo muestran que el número total y el porcentaje de exposición de los hogares y las viviendas a inundaciones y al calor extremo dentro de la RMs evidencian la existencia de grupos vulnerables frente a las amenazas climáticas y que este número aumentará en el largo plazo. En general, la exposición a inundaciones y al calor extremo para la RMs no se limita a los hogares con un determinado nivel socioeconómico o una particular condición física de la vivienda, sino que afecta a todas las categorías examinadas. Las personas más expuestas a las amenazas no se encuentran exclusivamente en los estratos socioeconómicos más bajos. Por el contrario, la comparación de la probabilidad de exposición muestra que los estratos más altos están más expuestos a fenómenos de inundación, mientras que los estratos más bajos son más vulnerables al calor extremo. Por tanto, es la amenaza lo que es diferente. No obstante, los patrones de distribución muestran un gran número de familias de estratos socioeconómicos medios y bajos que están expuestas a inundaciones y que necesitan medidas específicas de adaptación. Esta depende también de las experiencias previas con las inundaciones o el calor extremo, así como de las capacidades de recuperarse y adaptarse a ellos. Es decir, aunque los grupos socioeconómicos más altos están comparativamente más expuestos a las inundaciones, ello no significa que sufran más daños, puesto que cumplen con medidas de protección - por ej. muros de protección (Müller, 2012) - o están más equipados para protegerse y reaccionar a posibles 
daños (véase Kuhlicke et al., 2012). También en términos de exposición al calor extremo, sería interesante determinar si los patrones de distribución son el resultado de nuevos estilos de vida y de una elección deliberada para ganar prestigio social, en especial referencia a las viviendas de clase media y alta, que se han convertido -por ej. en España y California- en factores que determinan la exposición a esta amenaza (Romero-Calcerrada, Novillo, Millington \& Gómez-Jiménez, 2008). Por tanto, es necesario analizar con mayor profundidad la susceptibilidad y la capacidad de recuperación de la población afectada y las causas profundas de los patrones de exposición a las amenazas mediante la realización de estudios cualitativos a nivel local (Krellenberg et al., 2014).

Resumiendo, los resultados sugieren implementar distintas medidas de adaptación si se desea reducir la vulnerabilidad en la RMs. Las medidas de adaptación deberían considerar los patrones específicos de exposición a las amenazas. Es evidente que tales medidas deberían ser acordes a las estrategias generales de adaptación, por ej. las desarrolladas en la educación y la planificación urbana, e integradas en las políticas urbanas. Depende de las políticas de adaptación al cambio climático y del desarrollo urbano que se refuerce la capacidad para proteger a la población expuesta, disminuir su vulnerabilidad y, sobre todo, evitar un incremento de nuevas áreas expuestas.

\section{Conclusión}

El interés de este artículo se centró en indagar en las posiciones teóricas sobre los patrones de distribución de la vulnerabilidad, los que habitualmente se anticipan en el mundo académico. Estas posiciones se contrapusieron con evidencias empíricas derivadas de cálculos estadísticos. Nuestro análisis demuestra una conclusión destacable: no existe un patrón específico en la distribución espacial de la vulnerabilidad (exposición), sino que esta se encuentra determinada por la amenaza, afectando a todas las categorías de la dimensión socioeconómica, físico-constructiva y urbanorural. Hemos argumentado por qué se deberían verificar atentamente las vulnerabilidades frente a los impactos del cambio climático en la RMs.

Para la dimensión socioeconómica, la hipótesis de que los patrones de distribución de las vulnerabilidades están determinados por los estratos socioeconómicos más bajos, formulada por diferentes autores (por ej. Dasgupta, 1995; Cutter et al., 2000; Satterthwaite, Huq, Pelling, Reid \& Lankao, 2007), solo se confirma parcialmente para la RMS, donde la exposición a inundaciones y al calor extremo no se limita a un determinado estrato socioeconómico. Al contrario, los resultados indican que las familias acomodadas son de igual modo vulnerables a los impactos del cambio climático, por su lugar de residencia en zonas de alto riesgo de inundaciones, mientras que las familias de bajos recursos están más afectadas por el calor extremo. Al analizar la dimensión socioeconómica de la vulnerabilidad, se debería reconocer su variabilidad espacial, así como la sensibilidad con la cual los diferentes grupos de la sociedad anticipan y se preparan para estos impactos del cambio climático (Bohle, Downing \& Watts, 1994). Además, estas observaciones indican que en sociedades tan desiguales y estratificadas como las de América Latina, es muy probable que la susceptibilidad y la capacidad de recuperación desempeñen un 
papel importante. Eso va conforme con la nueva definición de la vulnerabilidad del Intergovernmental Panel on Climate Change (IPCC) (2012), que excluye la "exposición" como componente integral de la vulnerabilidad. Ello quizá porque la forma de disminuir la vulnerabilidad se limitó en muchos casos a reducir la exposición sin mejorar la calidad de vida.

En la dimensión físico-constructiva en los patrones de distribución de la vulnerabilidad, sostuvimos que es más probable que las viviendas con excelentes o valiosas condiciones físicas se encuentren en áreas de inundaciones, lo que difiere en el caso de la exposición al calor extremo. Las viviendas con condiciones físicas deficientes son las que muestran una elevada probabilidad de exposición. Aunque los nuevos proyectos inmobiliarios no necesariamente se desarrollan en áreas de exposición a amenazas, podrían intensificarlas e influir en su crecimiento a mediano y largo plazo. Se observan tendencias semejantes a las de la dimensión socioeconómica, donde el lugar de residencia influye en el grado de vulnerabilidad y la condición física de la vivienda no es el factor determinante.

Finalmente, en la dimensión urbano-rural, argumentamos que las diferencias entre la exposición a inundaciones y al calor extremo en las áreas urbanas y rurales difieren en un grado menor, por lo cual ambos tipos de áreas deberían ser adaptadas a los impactos del cambio climático en función de su contexto específico. A mediano y largo plazo es muy probable que todas las áreas, independientemente de que sean urbanas o rurales, experimenten un aumento en la exposición de su población y sus hogares. No obstante, esto dependerá de las políticas de adaptación al cambio climático y de la capacidad de recuperación de la sociedad urbana y rural. Aunque las áreas rurales ya han empezado a adaptarse a la variabilidad climática con mecanismos para enfrentarse a sequías e inundaciones, se ha observado pocos esfuerzos de adaptación en las áreas urbanas, posiblemente por la escasa conciencia de los gobiernos locales (Hardoy \& Pandiella, 2009).

Dado que la exposición al cambio climático afecta a todos los sectores de la sociedad y de la vida urbana, debería tratársela como una tarea política transversal a mediano y largo plazo. Siguiendo en esta línea, sería necesario que las estrategias políticas de adaptación al cambio climático reconocieran los elementos contextuales de las vulnerabilidades, que varían entre individuos y grupos sociales, así como entre territorios y a través del tiempo. Por ello, la vulnerabilidad al cambio climático no debería tratarse de forma estática, sino como una realidad dinámica que adopta diferentes formas y niveles (Handmer, Dovers \& Downing, 1999; Leichenko \& O’Brien, 2002; Eriksen, Brown \& Kelly, 2005; Thomas \& Twyman, 2005; Eriksen \& O’Brien, 2007). A través de los resultados obtenidos, se podría subrayar que el grado de vulnerabilidad que ya existe en la RMs invita a llevar a cabo acciones locales. Para minimizar el impacto de las amenazas por inundación y calor extremo, se requiere el desarrollo y la puesta en práctica de medidas de adaptación concretas. Es de suma importancia que se analice con profundidad todos los componentes de la vulnerabilidad. Las siguientes preguntas podrían resultar estimulantes: ¡cuál es el grado de preparación de los habitantes expuestos a amenazas hidroclimáticas? ¿Cómo se recuperarían ante catástrofes naturales? 


\section{Referencias bibliográficas}

Adger, W. N. \& Kelly, P. M. (1999). Social vulnerability to climate change and the architecture of entitlements. Mitigation and Adaptation Strategies for Global Change, 4(3-4), 253266. doi: 10.1023/A:1009601904210

Adger, W. N., Agrawala, S., Mirza, M. M. Q., Conde, C., O’Brien, K., Pulhin, J., ... Takahashi, K. (2007). Assessment of adaptation practices, options, constraints and capacity. En M. L. Parry, O. F. Canziani, J. P. Palutikof, C. E. Hanson \& P. J. van der Linden (Eds.), Climate change 2007: impacts, adaptation and vulnerability. Contribution of working group II to the Fourth Assessment Report of the Intergovernmental Panel on Climate Change (pp. 719-743). Cambridge: Cambridge University Press.

Ayala, L., Espinoza, G. \& Saragoni, R. (1987). Estudio de Áreas de Riesgo por Inundación. Segunda Parte. Santiago de Chile: Ministerio de Vivienda y Urbanismo.

Bankoff, G. (2001). Rendering the world unsafe: "Vulnerability" as western discourse. Disasters, 25(1), 19-35. doi: 10.1111/1467-7717.00159

Banzhaf, E., Kindler, A., Müller, A., Metz, K., Reyes-Paecke, S. \& Weiland, U. (2012). Landuse change, risk and land-use management. En D. Heinrichs, K. Krellenberg, B. Hansjürgens \& F. Martínez (Eds.), Risk Habitat Megacity (pp. 127-154). Heidelberg: Springer.

Bell, M. L., O’Neill, M. S., Ranjit, N., Borja-Aburto, V. H., Cifuentes, L. A. \& Gouveia, N. C. (2008). Vulnerability to heat-related mortality in Latin America: A case-crossover study in São Paulo, Brazil, Santiago, Chile and Mexico City, Mexico. International Journal of Epidemiology, 37(4), 796-804. doi: 10.1093/ije/dyn094

Birkmann, J., Cardona, O. D., Carreño, M. L., Barbat, A. H., Pelling, M., Schneiderbauer, S., ... Welle, T. (2013). Framing vulnerability, risk and societal responses: the MOVE framework. Natural Hazards 67(2), 193-211. doi: 10.1007/s11069-013-055

Bohle, H. G., Downing, T. E. \& Watts, M. J. (1994). Climate change and social vulnerability: Toward a sociology and geography of food insecurity. Global Environmental Change Volume, 4(1), 37-48. http://dx.doi.org/10.1016/0959-3780(94)90020-5

Burton, I., Kates, R. \& White, G. F. (1993). The environment as hazard (2a ed.). Nueva York: Guilford Press.

Cáceres, G. \& Sabatini, F. (Eds.). (2004). Barrios cerrados en Santiago de Chile: entre la exclusión y la integración residencial. Santiago de Chile: Lincoln Institute of Land Policy/Instituto de Geografía, Pontificia Universidad Católica de Chile (puc).

Chambers, R. (1989). Editorial Introduction: Vulnerability, coping and policy. IDS Bulletin, 20(2), 1-7. doi: 10.1111/j.1759-5436.1989.mp20002001.x

Cortés, G., Schaller, S., Rojas, M., García, L., Descalzi, A., Vargas, L. \& McPhee, J. (2012). Assessment of the current climate and expected climate changes in the Metropolitan Region of Santiago de Chile. Leipzig: UFz (Helmholtz Centre for Environmental Research) Report.

Cross, J. A. (2001). Megacities and small towns: Different perspectives on hazard vulnerability. Environmental Hazards, 3(2), 63-80. http://dx.doi.org/10.1016/S14642867(01)00020-1

Cutter, S. L., Boruff, B. J. \& Shirley, W. L. (2003). Social vulnerability to environmental hazards. Social Science Quarterly, 84(2), 242-261. En http://bit.ly/1j9Pa8X 
Cutter, S. L., Mitchell, J. T. \& Scott, M. S. (2000). Revealing the vulnerability of people and places: A case study of Georgetown County, South Carolina. Annals of the Association of American Geographers, 90(4), 713-737. doi: 10.1111/0004-5608.00219

Dasgupta, P. (1995). Population, poverty, and the local environment. Scientific American, 272(2), 40-45. doi: 10.1038/scientificamerican0295-40

Domènech, L., Supranamiam, M. \& Saurí, D. (2010). Droughts. Citizens' risk awareness and responses to the 2007-2008 drought episode in the Metropolitan Region of Barcelona (мкв). En G. Wachinger \& O. Renn (Eds.), Risk perception and natural hazards (pp. 47-56). CapHaz-Net, WP3. D3.1 - Version 3. Stuttgart.

Ebert, A., Welz, J., Heinrichs, D., Krellenberg, K. \& Hansjürgens, B. (2010). Socioenvironmental change and flood risks: The case of Santiago de Chile. Erdkunde, 64(4), 303-313. http://dx.doi.org/10.3112/erdkunde.2010.04.01

Eriksen, S., Brown, K. \& Kelly, P. M. (2005). The dynamics of vulnerability: Locating coping strategies in Kenya and Tanzania. Geographical Journal, 171(4), 287-305. doi: $10.1111 / j .1475-4959.2005 .00174 . x$

Eriksen, S.H. \&O'Brien, K. (2007). Vulnerability, poverty and the need for sustainableadaptation measures. Climate Policy, 7(4), 337-352. doi: 10.1080/14693062.2007.9685660

Feito, L. (2007). Vulnerabilidad. Anales de Sistema Sanitario de Navarra, 30(3), 7-22. En http:// scielo.isciii.es/scielo.php?pid=S1137-66272007000600002\&script=sci_abstract

Fuchs, S., Kuhlicke, C. \& Meyer, V. (2011). Editorial for the special issue: vulnerability to natural hazards - the challenge of integration. Natural Hazards, 58(2), 609-619. doi: $10.1007 / s 11069-011-9825-5$

González, D. \& Rodríguez, J. (2006). Redistribución espacial y migración interna de la población en Chile en los últimos 35 años (1965-2002): una síntesis de la hipótesis y la evidencia. Revista Estudios Demográficos y Urbanos, 21(2), 369-406.

Handmer, J. W., Dovers, S. \& Downing, T. E. (1999). Societal vulnerability to climate change and variability. Mitigation and Adaptation Strategies for Global Change, 4(3-4), 267-281.

Hardoy J. \& Pandiella, G. (2009). Urban poverty and vulnerability to climate change in Latin America. Environment and Urbanization, 21(1), 203-224.

Heinrichs, D., Krellenberg, K. \& Fragkias, M. (2013). Urban responses to climate change: Theories and governance practice in cities of the global South. International Journal of Urban and Regional Research, 37(6), 1865-1878. doi: 10.1111/1468-2427.12031

Heinrichs, D., Krellenberg, K., Hansjürgens, B. \& Martínez, F. (2011). Risk Habitat Megacity. Heidelberg: Springer.

Hewitt, K. (1997). Regions of risk: A geographical introduction to disasters. Essex, RU: Longman.

Hidalgo, R. (2004). De los pequeńos condominios a la ciudad vallada: las urbanizaciones cerradas y la nueva geografía social en Santiago de Chile (1990-2000). EURE, 30(91), 29-52. http://dx.doi.org/10.4067/S0250-71612004009100003

Höfer, R. (2013). Remote sensing based derivation of urban structure types to assess hydrometeorological impacts in highly dynamic urban agglomerations in Latin America (PhD dissertation 06/2013). Leipzig: Helmholtz Centre for Environmental Research (UFz). En https://www.freidok.uni-freiburg.de/data/9218

Instituto Nacional de Estadísticas (INE). (2002). Censos de Población y Vivienda 2002. Santiago de Chile. En http://www.ine.cl/canales/chile_estadistico/censos/censo_poblacion_ vivienda.php 
Intergovernmental Panel on Climate Change (IPCC). (2012). Managing the risks of extreme events and disasters to advance climate change adaptation. A Special Report of Working Groups I and II of the Intergovernmental Panel on Climate Change. [C. B. Field, V. Barros, T. F. Stocker, D. Qin, D. J. Dokken, K. L. Ebi, ... P. M. Midgley (Eds.)]. Nueva York: Cambridge University Press.

Janoschka, M. (2002). El nuevo modelo de la ciudad latinoamericana: Fragmentación y privatización. EURE, 28(85), 11-29. http://dx.doi.org/10.4067/S025071612002008500002

Jordán, R., Rehner, J. \& Samaniego, J. (2012). Megacities in Latin America: Role and challenges. En D. Heinrichs, K. Krellenberg, B. Hansjürgens. \& F. Martínez (Eds.), Risk habitat megacity (pp. 19-35). Heidelberg: Springer.

Kantor, P. \& Nair, P. (2005). Vulnerability among slum dwellers in Lucknow, India: Implications for urban livelihood security. International Development Planning Review, 27(3), 333358. doi: http://dx.doi.org/10.3828/idpr.27.3.4

Kaztman, R. \& Filgueira, C. (1999). Marco conceptual sobre activos, vulnerabilidad y estructura de oportunidades. [LC/MVD/R.176/Rev.1/E ]. Montevideo: Comisión Económica para América Latina y el Caribe (CEPAL). En http://www.cepal.org/publicaciones/ xml/6/10816/LC-R176.pdf

Krellenberg, K., Müller, A., Schwarz, A., Höfer, R. \& Welz, J. (2013). Flood and heat hazards in the Metropolitan Region of Santiago de Chile and the socio-economics of exposure. Applied Geography, 38, 86-95. http://dx.doi.org/10.1016/j.apgeog.2012.11.017

Krellenberg, K., Link, F., Welz, J., Harris, J., Barth, K. \& Irarrazaval, F. (2014). Supporting local adaptation: The contribution of socio-environmental fragmentation to urban vulnerability. Applied Geography, 55, 61-70. http://dx.doi.org/10.1016/j. apgeog.2014.08.013

Kuhlicke, C., Scolobig, A., Tapsell, S., Steinführer, A. \& de Marchi, B. (2011). Contextualizing social vulnerability: Findings from case studies across Europe. Natural Hazards, 58(2), 789-810. doi: 10.1007/s11069-011-9751-6

Kuhlicke, C., Kabisch, S., Krellenberg, K. \& Steinführer, A. (2012). Urban vulnerability under conditions of global environmental change. Conceptual reflections and empirical case examples of growing and shrinking cities. En S. Kabisch, A. Kunath, P. Schweizer-Ries \& A. Steinführer (Eds.), Vulnerability, Risks and Complexity: Impacts of Global Change on Human Habitats (pp. 27-38). Göttingen: Hogrefe.

Lafortezza, R., Carrus, G., Sanesi, G. \& Davies, C. (2009). Benefits and well-being perceived by people visiting green spaces in periods of heat stress. Urban Forestry and Urban Greening, 8(2), 97-108. http://dx.doi.org/10.1016/j.ufug.2009.02.003

Leichenko, R. \& O'Brien, K. (2002). The dynamics of rural vulnerability to global change. Mitigation and Adaptation Strategies for Global Change, 7(1), 1-18. En http://bit. ly/1OixvHf

Magrin, G., Gay García, C., Cruz Choque, D., Giménez, J. C., Moreno, A. R., Nagy, G. J., ... Villamizar, A. (2007). Latin America. Climate Change 2007: Impacts, adaptation and vulnerability. En M. L. Parry, O. F. Canziani, J. P. Palutikof, P. J. van der Linden \& C. E. Hanson (Eds.), Contribution of Working Group II to the Fourth Assessment Report of the Intergovernmental Panel on Climate Change (pp. 581-615). Cambridge: Cambridge University Press. 
McCarthy, J. J., Canziani, O. F., Leary, N. A., Dokken, D. J. \& White, K. S. (2001). Climate change 2001: Impacts, adaptation and vulnerability. Cambridge: Cambridge University Press.

Ministerio de Vivienda y Urbanismo (minvu). (2008). Propuesta de modificación del Plan Regulador Metropolitano de Santiago. Santiago: Autor.

Mitchell, J. K. (1999). Megacities and natural disasters: a comparative analysis. GeoJournal, 49(2), 137-142.

Müller, A. (2012). Areas at risk. Concept and methods for urban flood risk assessment. A case study of Santiago de Chile. Stuttgart: Franz Steiner Verlag.

Müller, A. \& Höfer, R. (2014). The impacts of climate and land-use change on flood and heat hazards. En K. Krellenberg \& B. Hansjürgens (Eds.), Climate Adaptation Santiago (pp. 107-126). Heidelberg: Springer.

Mysiak, J., Calliari, E., Carrera, L., Maziotis, A., Van Der Keur, P., Luther, J. \& Kuhlicke, C. (2012). CATALyst - Report on issues, gaps and opportunities, network coverage. Deliverable 2.2. En http://www.catalyst-project.eu/doc/dl_2-2.pdf

O’Brien, K., Leichenko, R., Kelkar, U., Venema, H., Aandahl, G., Tompkins, H., ... West, J. (2004). Mapping vulnerability to multiple stressors: climate change and globalization in India. Global Environmental Change, 14(4), 303-313. http://dx.doi.org/10.1016/j. gloenvcha.2004.01.001

O'Brien, K., Eriksen, S., Nygaard, L. \& Schjolden, A. (2007). Why different interpretations of vulnerability matter in climate change discourses. Climate Policy, 7(1), 73-88. doi: $10.1080 / 14693062.2007 .9685639$

Pelling, M. (2002). Assessing urban vulnerability and social adaptation to risk: evidence from Santo Domingo. International Development Planning Review, 24(1), 59-76. doi: http:// dx.doi.org/10.3828/idpr.24.1.4

Pelling, M. (1999). The political ecology of flood hazard in urban Guyana. Geoforum, 30(3), 249-261. http://dx.doi.org/10.1016/S0016-7185(99)00015-9

Pizarro, R. (2001). La vulnerabilidad social y sus desafios: una mirada desde América Latina. Serie Estudios estadísticos y prospectivos N ${ }^{\circ} 6$ (LC/L.1490-P). Santiago: Comisión Económica para América Latina y el Caribe (CEPAL), División de Estadística y Proyecciones Económicas. En http://bit.ly/1ODdmcR

Pizarro Hofer, R. (1999). Vulnerabilidad social en América Latina y el Caribe. Santiago: Centro Latinoamericano y Caribeño de Demografía (CELADE).

Puente, S. (1999). Social vulnerability to disaster in Mexico City. En J. K. Mitchell (Ed.), Crucibles of hazard: Mega-cities and disasters in transition (pp. 295-334). Tokyo: United Nations University Press.

Romero, H. \& Vásquez, A. (2005). Evaluación ambiental del proceso de urbanización de las cuencas del piedemonte andino de Santiago de Chile. EURE, 31(94), 97-118. http:// dx.doi.org/10.4067/S0250-71612005009400006

Romero, H., Moscoso, C. \& Smith, P. (2009). Lecciones y conclusiones sobre la falta de sustentabilidad ambiental del crecimiento espacial de las ciudades chilenas. En R. Hidalgo, C. de Mattos \& F. Arenas (Eds.), Chile del país urbano al país metropolitano (pp. 89-110). Santiago: Serie GEOlibros/EURE-Libros. 
Romero-Calcerrada, R., Novillo, C. J., Millington, J. D. A. \& Gómez-Jiménez, I. (2008). GIS analysis of spatial patterns of human-caused wildfire ignition risk in the sw of Madrid (Central Spain). Landscape Ecology, 23(3), 341-354. doi: 10.1007/s10980-008-9190-2

Romero Lankao, P. (2010). Water in Mexico City: What will climate change bring to its history of water-related hazards and vulnerabilities? Environment and Urbanization, 22(1), 157-178. doi: 10.1177/0956247809362636

Ruiz-Tagle, J. \& López, E. (2014). El estudio de la segregación residencial en Santiago de Chile: revisión crítica de algunos problemas metodológicos y conceptuales. EURE, 40(119), 25-48. http://dx.doi.org/10.4067/S0250-71612014000100002

Sabatini, F. (2000). Reforma de los mercados de suelo en Santiago, Chile: efectos sobre los precios de la tierra y la segregación residencial. EURE, 26(77), 49-80. http://dx.doi. org/10.4067/S0250-71612000007700003

Sabatini, F., Salcedo, R., Wormald, G. \& Cáceres, G. (Eds.). (2010). Tendencias de la segregación en las principales ciudades chilenas. Santiago: Instituto Nacional de Estadísticas (INE).

Sabatini, F., Cáceres, G. \& Cerda, J. (2001). Segregación residencial en las principales ciudades chilenas: Tendencias de las tres últimas décadas y posibles cursos de acción. EURE, 27(82), 21-42. http://dx.doi.org/10.4067/S0250-71612001008200002

Satterthwaite, D., Huq, S., Pelling, M., Reid, H. \& Lankao, P. R. (2007). Adapting to climate change in urban areas. The possibilities and constraints in low-and middle-income nations. Londres: International Institute for Environment and Development.

Swyngedouw, E., Kaïka, M. \& Castro, E. (2002). Urban water: A political-ecology perspective. Built Environment, 28(2), 124-137. En http://eprint.ncl.ac.uk/pub_details2. aspx?pub_id=47331

Thomas, D. S. G. \& Twyman, C. (2005). Equity and justice in climate change adaptation amongst natural-resource-dependent societies. Global Environmental Change, 15(2), 115-124. http://dx.doi.org/10.1016/j.gloenvcha.2004.10.001

Uitto, J. I. (1998). The geography of disaster vulnerability in megacities: A theoretical framework. Applied Geography, 18(1), 7-16. http://dx.doi.org/10.1016/S01436228(97)00041-6

Walker, G., Burningham, K., Fielding, J., Smith, G., Thrush, D. \& Fay, H. (Eds.). (2006). Addressing environmental inequalities. Flood risk. Bristol: Environment Agency.

Ward, P. M. (1998). Mexico City (2a ed.). Chichester, Nueva York: John Wiley and Sons.

Welz, J. (2014). Segregation und Integration in Santiago de Chile zwischen Tradition und Umbruch. Stuttgart: Franz Steiner Verlag.

Welz, J., Schwarz, A. \& Krellenberg, K. (2014). Understanding hazard exposure for adaptation in a climate change context. En K. Krellenberg \& B. Hansjürgens (Eds.), Climate Adaptation Santiago (pp. 127-147). Heidelberg: Springer.

Winchester, L. (2008). La dimensión económica de la pobreza y precariedad urbana en las ciudades latinoamericanas. Implicaciones para las políticas del hábitat. EURE, 34(103), 27-47.

Winchester, L. \& Szalachman, R. (2012). The urban poor's vulnerability to climate change in Latin America and the Caribbean. En D. Hoornweg, M. Freire, M. J. Lee, P. BhadaTata \& B. Yuen (Eds.), Cities and the urgent challenges of climate change: introduction (pp. 727-751) (Vol. 2 de Cities and climate change: responding to an urgent agenda. Urban development series). Washington D.c.: The Worldbank. 
Wisner, B., Blaikie, P., Cannon, T. \& Davis, I. (2004). At risk. Natural hazards, people's vulnerability and disasters. Londres: Routledge.

Wisner, B. (1998). Marginality and vulnerability: Why the homeless of Tokyo don't "count" in disaster preparations. Applied Geography, 18(1), 25-33. http://dx.doi.org/10.1016/ S0143-6228(97)00043-X 